\title{
COMPARACION DE TAXOCENOSIS DE SALTAMONTES (Orth., Caelifera) EN DISTINTOS SISTEMAS MONTAÑOSOS DEL MEDITERRÁNEO OCCIDENTAL
}

\author{
Por \\ M. ${ }^{a}$ D. García ${ }^{1} ;$ J.J. Presa ${ }^{1}$ y L. Ramírez-Díaz ${ }^{2}$ \\ COMPARATIVE STUDY OF GRASSHOPPERS‘ TAXOCENOSIS \\ (Orth., Caelifera) ON VARIOUS MOUNTAINS \\ OF THE WEST MEDITERRANEAN REGION
}

\section{SUMMARY}

Grasshopers' taxocenosis of nine mountains of the west Mediterranean region are compared using factorial correspondence analysis.

Results suggest that mountains can be grouped according to the fauna of areas wich are separated by biogeographical barriers: north-african mountains, iberian mountains and eurosiberian mountains. In each group, different mountains are discriminated by their peculiar and characteristic species.

\section{RESUMEN}

Se comparan las taxocenosis de saltamontes de nueve sistemas montañosos del Mediterráneo Occidental: Cazorla y Segura, Espuña, Sierra Nevada, Guadarrama, Pirineos, Macizo Central, Alpes, Apeninos y Atlas, utilizando el análisis factorial de las correspondencias. Los resultados indican que los sistemas montañosos se agrupan en función de la fauna perteneciente a las áreas separadas por barreras biogeográficas importantes: Atlas, sistemas ibéricos y sistemas eurosiberianos. Dentro de cada grupo, los distintos sistemas montañosos se diferencian por las especies que les son propias y características.

\section{INTRODUCCION}

Un aspecto relevante en ecología, al que no se ha prestado excesiva atención, se refiere al estudio comparativo de características estructurales y funcionales de comunidades de organismos entre distintas zonas geográficas (AGUILAR AMAT, 1981; HERRERA, 1980; SANTOS, 1983, THIELE, 1977).

(1) Departamento de Zoología. Facultad de Bilogia. Universidad de Murcia.

(2) Departamento de Ecologia. Facultad de Biologia. Universidad de Murcia. 
A este respecto, resulta de interés conocer la relación que existe entre la fauna acridológica de un sistema montañoso dado y otros, próximos o relacionados en cierta medida desde el punto de vista del medio físico, presuponiendo, no obstante, que las grandes elevaciones de terreno, más o menos aisladas, presentan una variabilidad semejante a la de un archipiélago (ALEXANDER \& ILLIARD, 1969). No obstante, los saltamontes tienden a ser abundantes pero no muy móviles, por lo que las diferencias respecto a distribución en distancias geográficas pequeñas pueden resultar particularmente significativas (CLARIDGE \& SINGHRAO, 1978).

En este trabajo se pretende localizar la fauna acridológica de Sierra Espuña dentro de un grupo de sistemas montañosos de los países de la cuenca occidental del Mediterráneo, teniendo en cuenta que la fauna de las montañas españolas resulta interesante porque los elementos mediterráneos ascienden, en ellas, a las mayores alturas, produciendo cierto número de endemismos o formas localizadas, incluso, en una sola montaña (UVAROV, 1928).

La comparación entre la fauna de acrídicos de distintos sistemas montañosos no ha sido objeto de ningún trabajo, si se exceptúa la realizada por PRESA, 1978, con las especies comunes de la Sierra del Guadarrama, Apeninos, Alpes y Pirineos.

\section{MATERIAL Y METODOS}

Para el estudio comparativo se consideran, además de Sierra Espuña, otras tres sierras ibéricas: Cazorla y Segura, Guadarrama y Nevada; un sistema montañoso de gran importancia biogeográfica: Pirineos; tres sistemas montañosos que pueden presentar diferencias apreciables con los anteriores debido a su lejanía, no a su latitud y clima: Macizo Central francés, Alpes y Apeninos y, por último, el Atlas, importante sistema montañoso del norte de Africa, área geográfica cuya fauna tiene afinidades con la del Sur de España (BURR, 1900).

Lo ideal sería poder comparar no sólo listas faunísticas, sino también la fenología y otros aspectos estructurales y funcionales que muestra cada especie en cada sistema montañoso, pero no es posible porque, o la información que se posee es fragmentaria, a falta de un estudio global del sistema montañoso (caso del Atlas y Apeninos) o, en caso de existen- 
cia de tal estudio global, no se contempla en él el estudio fenológico y ecológico de las especies. Este aspecto resulta conocido únicamente en las Sierras de Guadarrama y Espuña.

Los datos se han recogido de diferentes autores: BACCETTI, 1963: CERRO, 1980; CHAPMAN, 1937, 1938; CHOPARD, 1939, 1943; DREUX, 1961; GALVAGNI, 1959; GARCIA, 1983, MARTY, 1969; PASCUAL, 1978; PRESA, 1978; VOISIN, 1979; WERNER, 1934. Se ha contabilizado un total de 151 especies.

La relación de especies y su presencia en las diferentes Sierras se expone a continuación en forma de matriz de datos binarios, donde las letras que encabezan cada columna y sus equivalencias son:

CZ.- Sistema de Sierras de Cazorla y Segura (provincia de Jaén, España).

ES.- Sierra de Espuña (provincia de Murcia, España).

GD. - Sierra de Guadarrama (provincias de Avila, Madrid y Segovia, España).

SN. - Sierra Nevada (provincia de Granada, España).

PI.- Cordillera de los Pirineos (límite entre España y Francia).

MC.- Macizo Central francés (Francia, región central).

AL.- Cordillera de los Alpes (Sureste de Francia).

AP.- Cordillera de los Apeninos (Italia).

AT.- Cordillera del Atlas (Marruecos).

El nombre de las especies aparece completo y en el mismo hay tres letras en negritas que, leídas correlativamente, forman el código de la especie, con el que aparece en la Figura 1. En esta figura, igualmente, aparecen los diferentes sistemas montañosos con sus letras clave.

La tabla de datos se resumió mediante el uso del análisis factorial de las correspondencias (BENZECRI et al., 1973; CORDIER, 1965). 

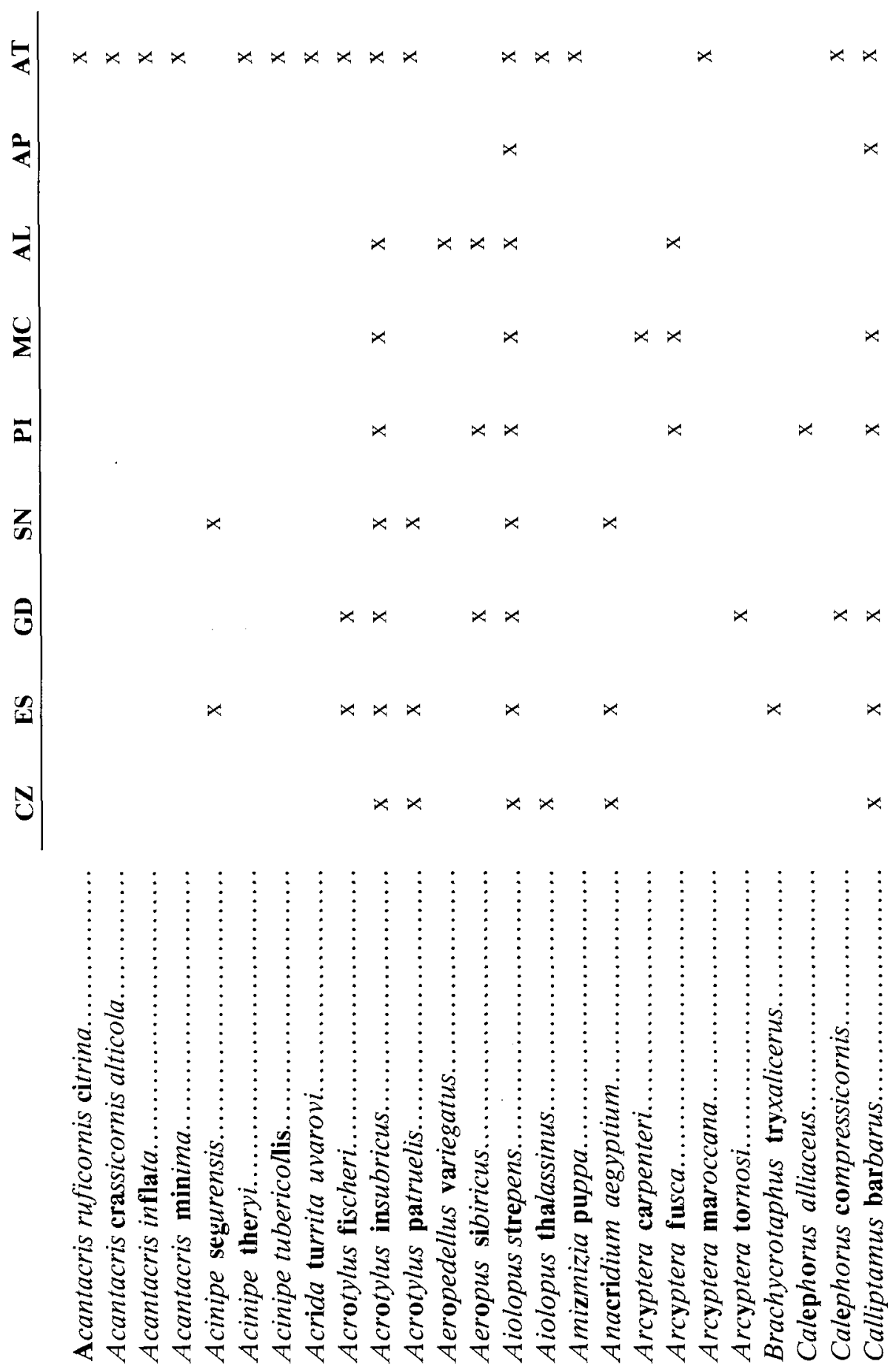


\begin{tabular}{|c|c|c|c|c|c|c|c|c|c|c|c|c|c|c|c|c|c|c|}
\hline$x$ & $x$ & $x$ & $x$ & & $x$ & $x$ & & $x$ & & & & & $x$ & & & & & \\
\hline & & & & & & & & & & $x$ & $x ;$ & $x$ & & $\infty$ & $x$ & & & $x$ \\
\hline$x$ & & $\ltimes$ & & $x$ & $x$ & $x$ & & & & & $x$ & & & $x$ & $x$ & & & $x$ \\
\hline$x$ & $x$ & $x$ & & $x$ & & $x$ & & $x$ & $x$ & $x$ & $x$ & & & & $x$ & $x$ & $x$ & $x$ \\
\hline$x$ & $x$ & $x$ & $x$ & $x$ & $x$ & & & $x$ & $x$ & & & & $x=$ & $x$ & & $x$ & & $x$ \\
\hline$x$ & $x$ & & & $x$ & & & & $x$ & & $x$ & & & $x$ & & & $x$ & $x$ & $x$ \\
\hline$x x$ & $x$ & & $x$ & & & $x$ & $x$ & $x$ & & & & $x$ & $x$ & & & & $x$ & $x$ \\
\hline$x$ & $x$ & & $x$ & & & & $x$ & $x$ & & & & & $x$ & & & & & \\
\hline$x$ & $x$ & & & & & & & & & $x$ & $x$ & & $x$ & & & & $x$ & \\
\hline
\end{tabular}

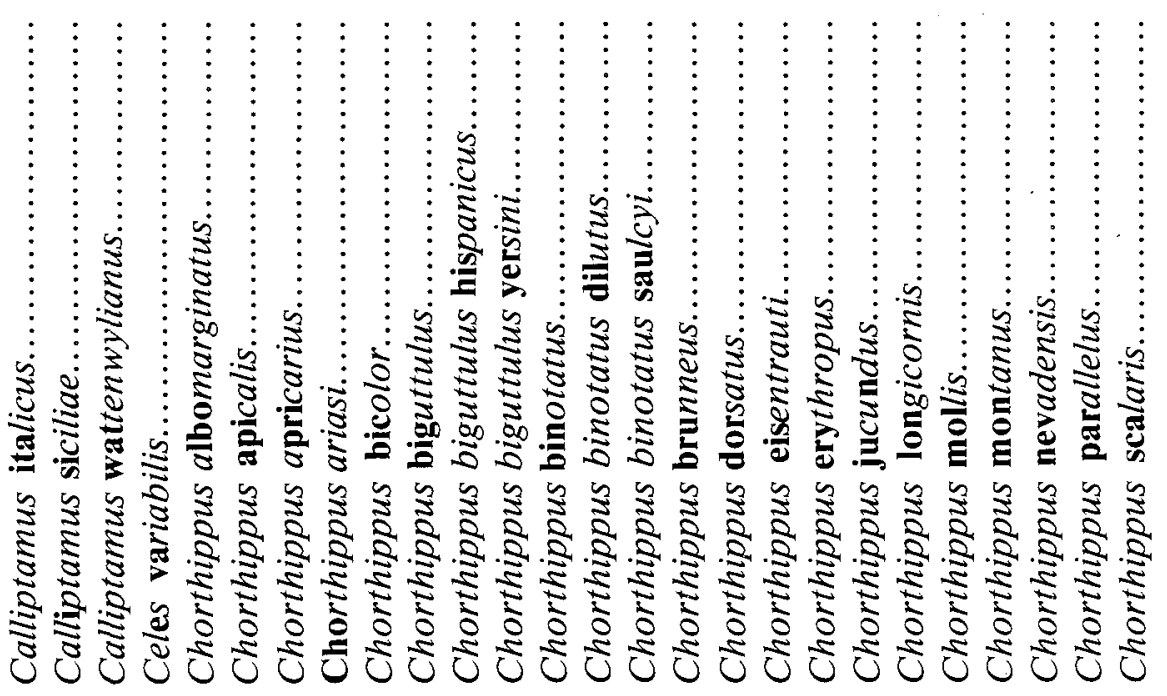



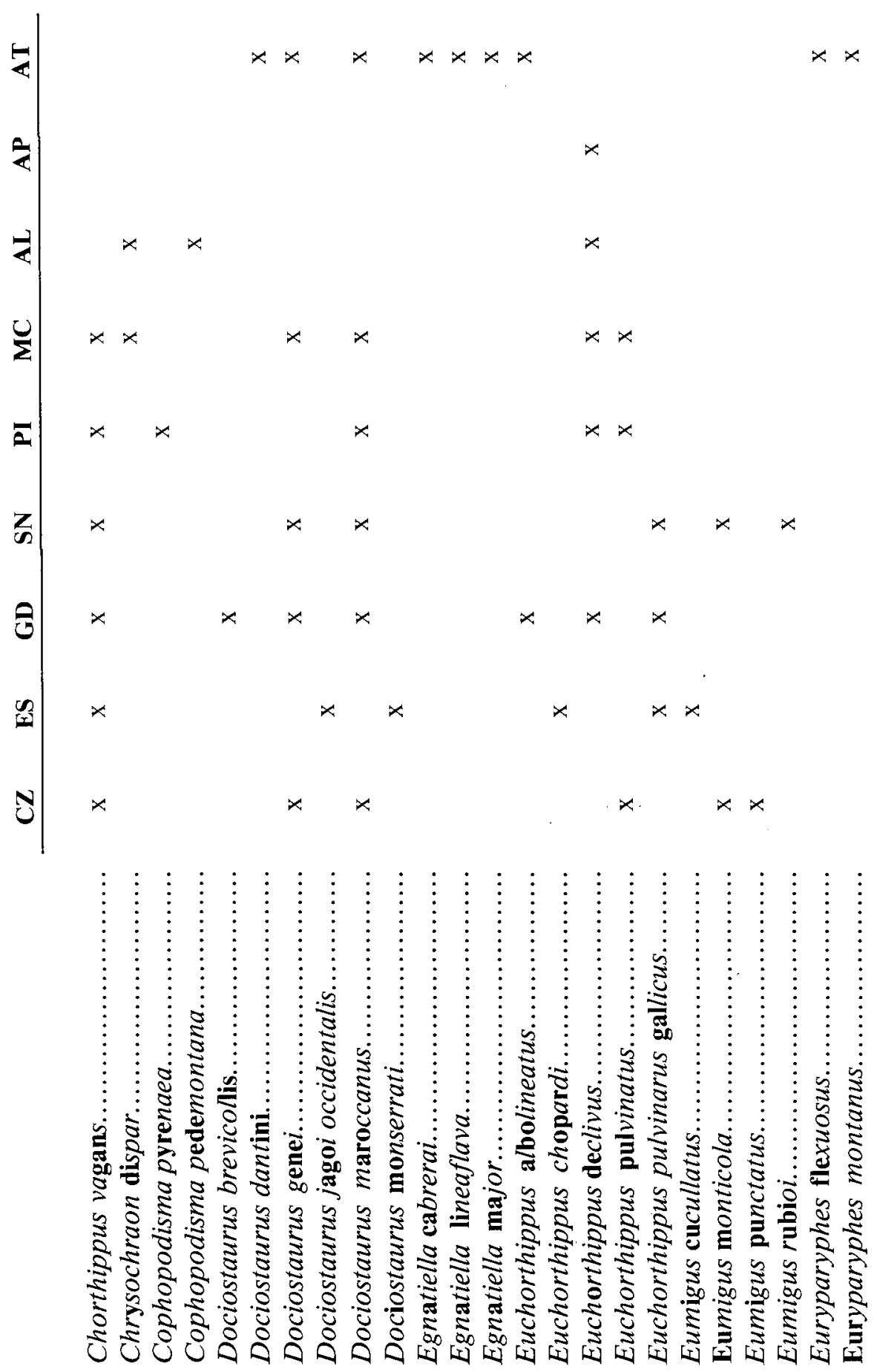


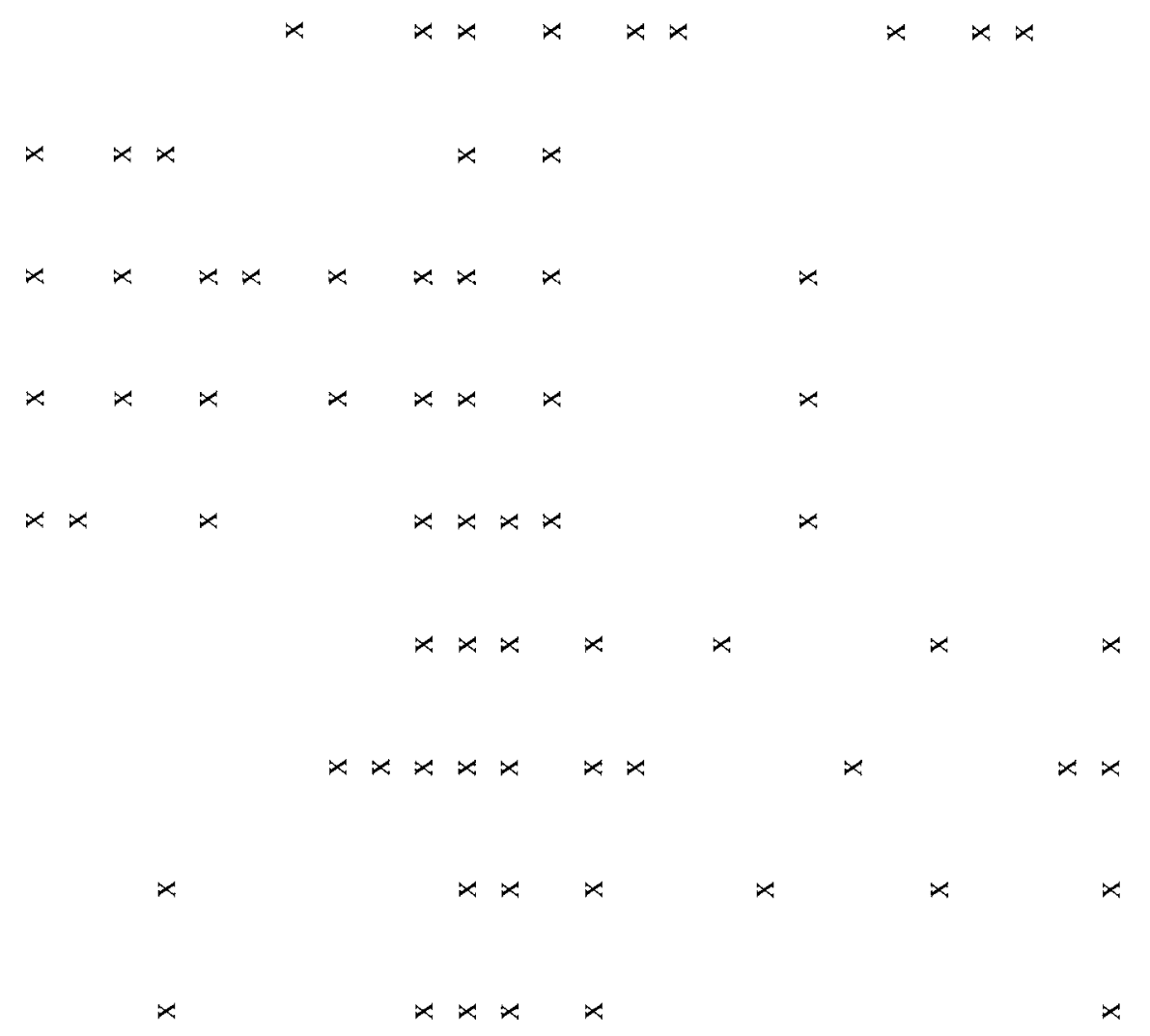

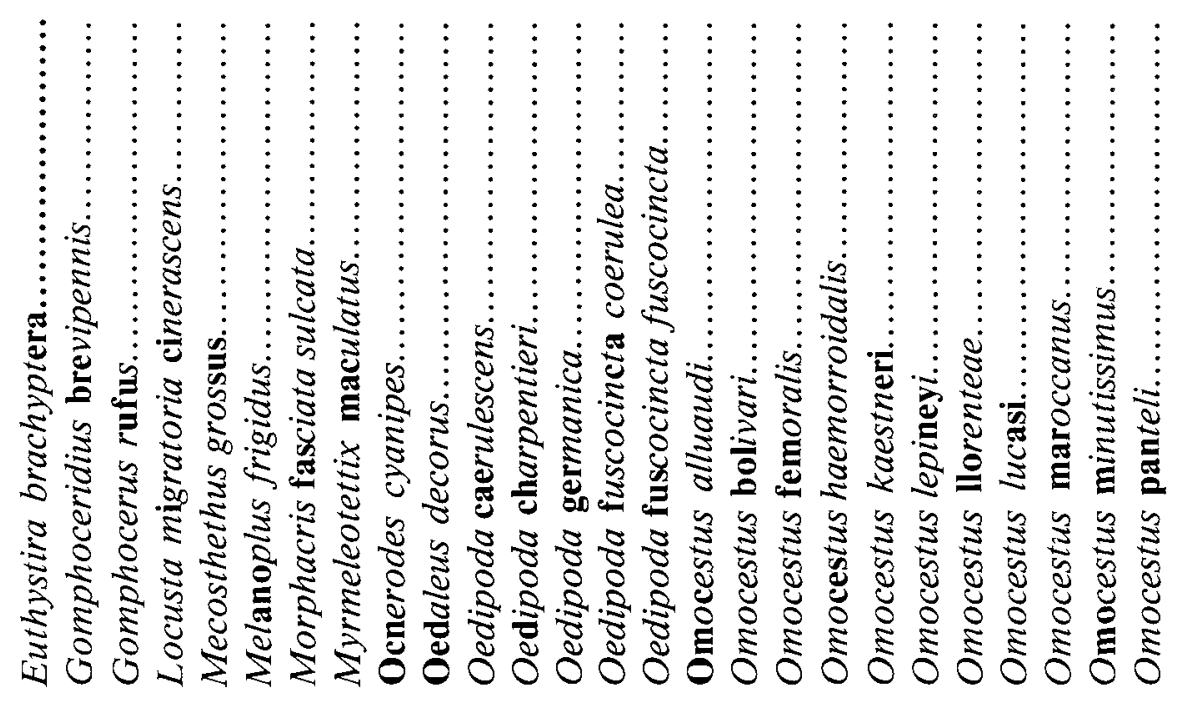



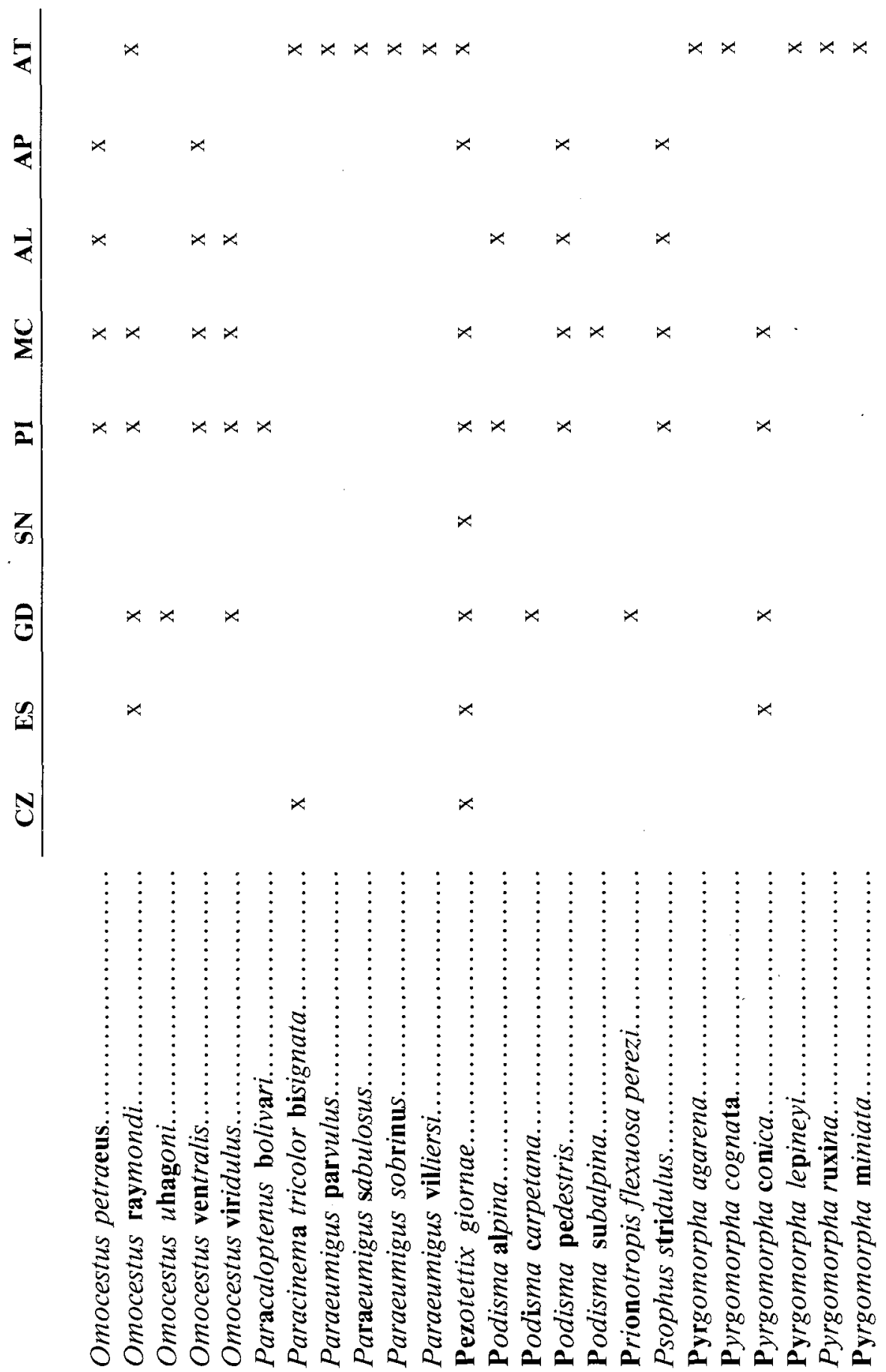

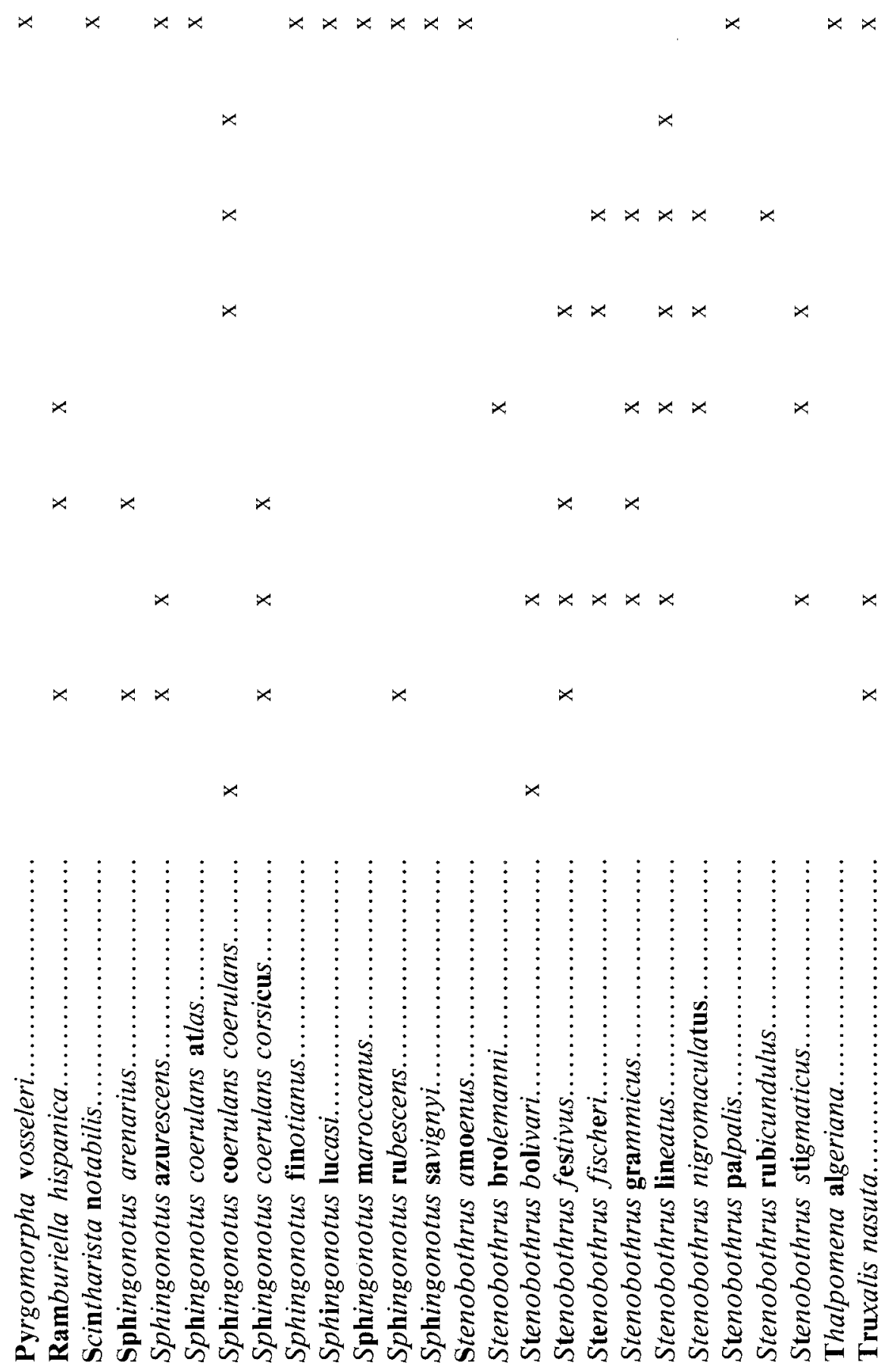

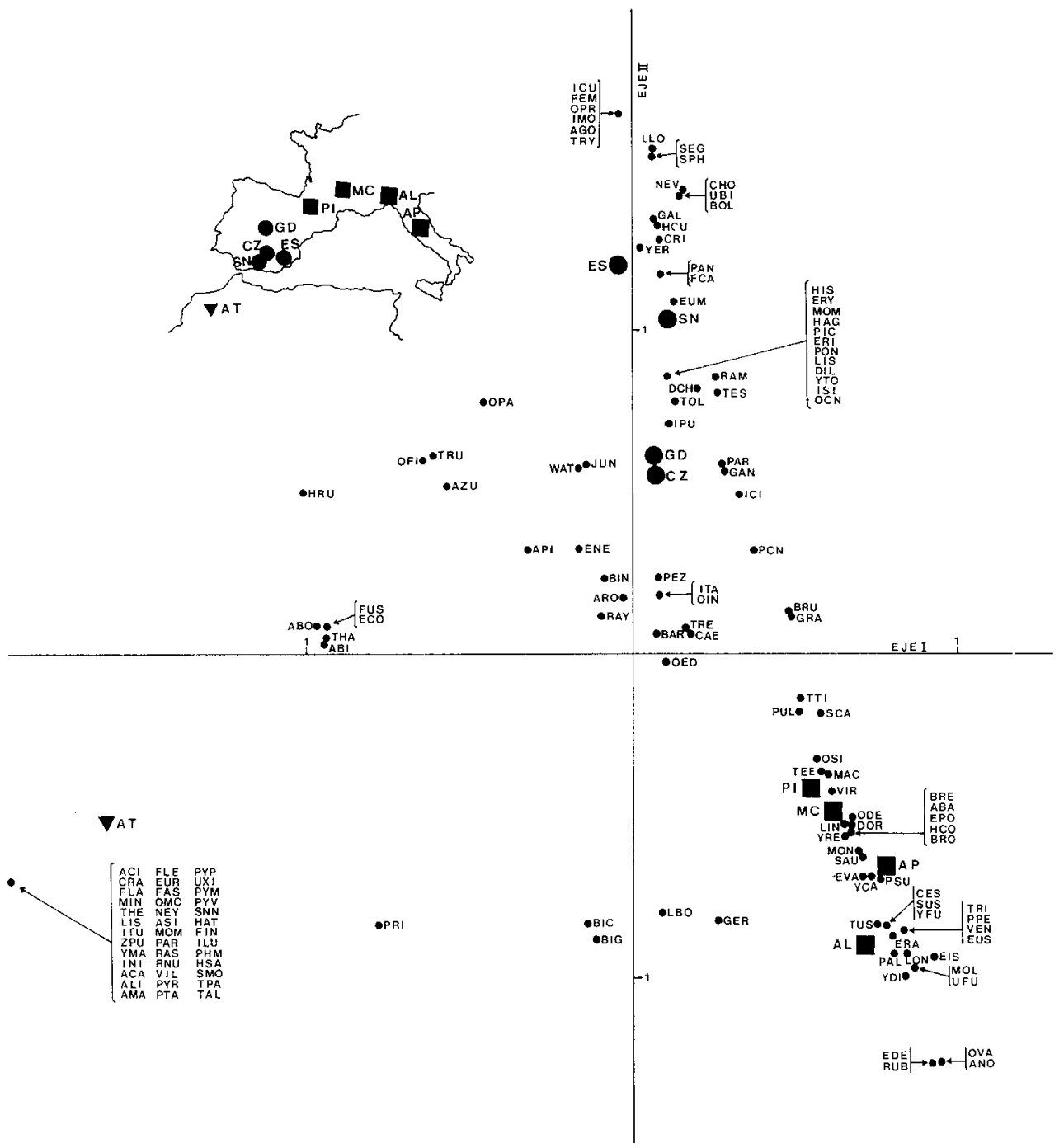

Figura 1.- Representación gráfica de los resultados del análisis factorial de las correspondencias de la matriz de 9 sistemas montañosos x 151 especies, en el plano definido por los ejes I y II. 


\section{RESULTADOS}

Se analizaron los datos a partir de una matriz de dimensiones 9 sistemas montañosos por 151 especies, la cual se sometió a un análisis factorial de correspondencias. El análisis proporcionó 5 ejes, cuyas varianzas absorbidas fueron:

$\begin{array}{cccccc}\text { I } & \text { II } & \text { III } & \text { IV } & \text { V } & \text { Total } \\ 26,91 \% & 21.70 \% & 13.61 \% & 10.23 \% & 8.62 \% & 81.07 \%\end{array}$

De las representaciones gráficas de los planos en los distintos ejes, la que resultó más interpretable fue la de los ejes I y II, cuyas varianzas absorbidas, además, son las más elevadas.

Esta representación compone la Figura 1, donde aparecen los diferentes sistemas montañosos considerados agrupados en función de las correspondencias entre las especies presentes y ausentes en cada uno de ellos.

Así, el Atlas aparece completamente desligado del resto y caracterizado por una extensa lista de especies exclusivas de él, en la parte negativa del eje I.

En la parte positiva del eje II aparecen las cuatro sierras ibéricas, apareciendo con el mayor valor Sierra Espuña y con el menor el sistema de Sierras de Cazorla y Segura.

En la parte negativa del mismo eje aparecen los demás sistemas montañosos considerados, estando más próximo al origen de coordenadas los Pirineos, y más alejados los Alpes.

Así y, con independencia del Atlas, aparecen dos grupos de sistemas montañosos bien definidos, el superior, conformado por las sierras estrictamente ibéricas, de fauna claramente mediterránea y, el inferior, de sistemas montañosos cuya fauna acridológica es, fundamentalmente, eurosiberiana. 


\section{DISCUSION Y CONCLUSIONES}

Según los resultados anteriores, la Sierra de Espuña es el sistema montañoso más aislado biogeográficamente dentro de los sistemas montañosos europeos. Sin duda se debe a su menor elevación y a su particular posición geográfica, que le confieren una climatología peculiar. Cercana a ella se encuentra Sierra Nevada que, aunque de mayor elevación, resulta próxima, biogeográficamente, a Sierra Espuña.

La Sierra de Guadarrama aparece más próxima al grupo inferior, pues su posición geográfica y su vegetación también lo son y, a la vez, constituye una barrera biogeográfica importante para algunos elementos europeos que han penetrado en la Península.

Resulta sorprendente, sin embargo, la posición que ocupa el sistema de Sierras de Cazorla y Segura puesto que, tanto su altitud como su posición geográfica deberían colocarlo en las proximidades de Sierra Nevada y Sierra Espuña, bien alejados del conjunto de sistemas montañosos eurosiberianos.

La explicación a esta posición debe buscarse en la no menos extraña lista faunística que aporta CERRO, 1980, en su trabajo. Esta resulta deficiente en la determinación de ciertos taxones y presumiblemente errónea en la determinación de otros, no escasos precisamente. A pesar de esto $\mathrm{y}$, al no poder haber estudiado los ejemplares y confirmado, o no, su determinación, se debe dar por buena la lista faunística, aunque se debe dejar constancia de las dudas que ofrece.

Ya en el grupo inferior, los Pirineos son el sistema montañoso más próximo a los estrictamente ibéricos, posición ésta que resulta intuitiva y pone de manifiesto, una vez más, el papel que ejerce como barrera biogeográfica entre el resto de Europa y la Península Ibérica.

Próximo a él aparece el Macizo Central francés que, aunque con un buen número de especies básicamente eurosiberianas, alberga ciertos elementos mediterráneos, termófilos, que justifican esta localización.

La posición de los Apeninos puede deberse a dos razones: lo fragmentario e incompleto del conocimiento de su fauna y su posición latitudinal, más semejante a la Península Ibérica que a la de los sistemas mon- 
tañosos continentales considerados. De no ser por ello, en buena lógica, debido al aislamiento biogeográfico, su posición sería mucho más aislada y alejada del origen de coordenadas.

Los Alṇes se presentan como los más alejados respecto a los sistemas montañosos ibéricos. Su fauna es muy diferente. Abundan los elementos de altura y escasean los elementos mediterráneos termófilos. $\mathrm{Su}$ gran elevación y sus consiguientes climatología y tipos de vegetación, inducen una fauna bastante distinta a las anteriores, a pesar de no estar muy alejadas geográficamente.

En cuanto al Atlas, a pesar de estar localizado, como ya se ha indicado, en una zona con afinidades faunísticas con el Sur de España, se encuentra muy alejado y aislado. Esto se debe, sin duda, a la gran extensión que ocupa este sistema montañoso y a la gran elevación que alcanza, lo que induce la aparición de buen número de formas propias. El tipo de clima presente en esa zona favorece la existencia y proliferación de los Acrídicos, por lo que el número de especies resulta elevado. Además, no hay que olvidar la barrera biogeográfica que constituye el estrecho de Gibraltar, que tiene como consecuencia la clara diferenciación de dos faunas acridológicas: europea y africana, cuyas especies comunes son aquéllas que ocupaban toda la cuenca mediterránea antes de la separación.

Todos los resultados abundan en las ideas de ALEXANDER \& HILLIARD, 1969 y CLARIDGE \& SINGHRAO, 1978, de considerar cada sistema montañoso de cierta envergadura como aislado y diferente faunísticamente del entorno y de otros sistemas próximos.

Sin embargo, la presencia de barreras biogeográficas lleva a la aparición de faunas propias de las regiones delimitadas por estas barreras. Todas las subregiones comprendidas en ella poseen una fauna común, la propia de la región, y se diferenciarán entre sí por la posesión de elementos faunísticos propios y característicos de cada subregión.

En la figura 1 aparecen tres grupos de sistemas montañosos: el norteafricano, el ibérico y el eurosiberiano, diferenciados por un tipo de fauna y, dentro de ellos, cada sistema montañoso aparece tipificado por sus especies características. 


\section{BIBLIOGRAFIA}

AGUILAR AMAT, J., (1981). Descripción de la comunidad de patos del Parque Nacional de Doñana. Doñana. Acta Vertebrata, 8: 125-128.

ALEXANDER, G. \& HILLIARD, J.R., (1969). Altitudinal and Seasonal Distribution of Orthoptera in the Rocky Mountains of Northern Colorado. Ecological Monographs, 39, n. ${ }^{\circ} 4: 385-$ 431.

BACCETTI, B., (1963). Notulae Orthopterologicae Xix. Ricerche sugli Ortotteroidei dell'Appennino ligure orientale per il Centro di Entomologia alpina e Forestale del C.N.R. Redia, 48: 93-163.

BENZECRI, J.P. et al., (1973). L'analyse des données. II. L'analyse des correspondences. Dunod Paris.

BURR, M., (1900). On the geographical distribution of European Orthoptera. Ent. Rec., 12: 47-48.

CERRO, A. del, (1980). Catálogo sistemático de los Orthopteroidea (sensu lato) del Coto Nacional de las Sierras de Cazorla y Segura. En: Viedma: Fauna de Cazorla. Invertebrados: 109-114. Monografias del Ministerio de Agricultura n. ${ }^{\circ} 23$. ICONA.

CLARIDGE, M.F. \& SINGHRAO, J.S.; (1978). Diversity and altitudinal distribution of grasshoppers (Acridoidea) on a Mediterranean mountain. Journ. Biogeography, 5: 239-250.

CORDIER, B., (1965). Analyse factorielle des correspondences. Thèse. $3 .^{\circ}$ cycle. Fac. Sci. Ronnes. $65 \mathrm{pp}$.

CHAPMAN, K.H., (1937). A new grasshopper (Orthoptera, Acrididae) from the Middle Atlas mountains, Morocco. Ent. Monthly Mag., 73: 127-129.

CHAPMAN, K.H., (1938). Orthoptera collected in the Atlas mountains Morocco. 1934-1936. Part I. Proc. R. ent. Soc. London (B), 7: 89-102.

CHOPARD, L., (1939). Récoltes de R. Paulian et A. Villiers dans le Haut Atlas marocain, 1938 (Quatrième note). Dictyoptères, Orthoptères et Dermaptères. Bull. Soc. Sc. nat. maroc., 19: 107-115.

CHOPARD, L., (1943). Faune de l'Empire français. I. Orthoptéroïdes de l'Afrique du Nord. Larose. Paris. 450 pp.

DREX, Ph., (1961). Recherches écologiques et biogeographiques sur les Orthoptères des Alpes françaises. Ann. Sci. Naturelles Zool. 12. ${ }^{\text {a }}$ 5., 3: 325-760.

GALVAGNI, A., (1959). Studio ecologico sistematico suglo Ortotteroidei dei Monti Sibillini. Mem. Mus. Stor. nat. Verona, 7: 1-76.

GARCIA, M.D., (1983). Estudio faunistico y ecológico de los Acridoidea (Orth., Insecta) de Sierra Espuña (Murcia). Tesis Doctoral. Universidad de Murcia. $284 \mathrm{pp.}$

HERRERA, C.M., (1980). Composición y estructura de dos comunidades mediterráneas de Passeriformes. Doñana Acta Vertebrata, VII (4). 340 pp. (Número especial). 
MARTY, R., (1969). Ecologie et Biocénotique des Orthoptères des Pyrénées. Vie et Milieu 20 (2c): 65-169.

PASCUAL, F., (1978). Estudio preliminar de los Ortópteros de Sierra Nevada, I: Introducción general e inventario de las especies. Bol. Asoc. esp. Entom., 1: 163-175.

PRESA, J.J., (1978). Los Acridoidea (Orthoptera) de la Sierra del Guadarrama. Trab. Cát. Artróp. Madrid, n. ${ }^{\circ} 26.277 \mathrm{pp}$.

SANTOS, A. de los, (1983). Biología y ecología de dos comunidades mediterráneas de Coleópteros terrestres de superficie. Tesis Doctoral. Universidad de Murcia. $332 \mathrm{pp}$.

THIELE, H.V., (1977). Carabid beetles in their environments. Heidelberg. Springer Verlag. New York. 369 pp.

UVAROV, B.P., (1928). Orthoptera of the Mountains of the Paleartic Region. Soc. Biogéographie. Paris, 2: $135-141$.

VOISIN, J.F., (1979). Autoécologie et biogéographie des Orthoptères du Massif Central. Tesis Doctoral. Université Pierre et Marie Curie. Paris. 354 pp.

WER NER, F., (1934). Deux nouvelles sauterelles du Moyen Atlas. Bull. Soc. Sci. Nat. Maroc, 14: 51-53. 